\title{
Paxillin functions as an oncogene in human gliomas by promoting cell migration and invasion
}

This article was published in the following Dove Press journal:

OncoTargets and Therapy

9 November 2016

Number of times this article has been viewed

\section{Bing Chen \\ Lei Xia \\ Chang-song $\mathrm{Xu}$ \\ Feng Xiao \\ Yan-feng Wang}

Department of Neurology, Huai'an First People's Hospital, Nanjing Medical University, Huai'an, People's Republic of China
Correspondence: Yan-feng Wang Department of Neurology, The First Hospital of Huai'an City Affiliated to Nanjing Medical University, \#6 Beijing West Road, Huaiyin District, Huai'an, Jiangsu 223300, People's Republic of China

Tel +86 I35 I524 8I80

Email758565376@qq.com
Background: Paxillin is implicated in tumorigenesis, progression and aggressive phenotypes of various malignancies, highlighting its functions in cellular adhesion, migration and survival. However, the roles of paxillin in human gliomas remain unclear. The aim of this study was to evaluate the clinical implication of paxillin expression in patients with gliomas and its biological function in glioma cells.

Patients and methods: Expression levels of paxillin gene and protein, respectively, were detected by quantitative real-time reverse transcription polymerase chain reaction, Western blot and immunohistochemistry analyses in 120 pairs of glioma and matched nontumorous brain tissues. The associations between paxillin expression and various histopathological features of glioma patients were also statistically evaluated. Then, the functions of paxillin in cell migration and invasion of glioma cell lines were determined by transwell assays in vitro.

Results: The expression levels of both paxillin gene and protein in glioma tissues were markedly higher than those in matched nontumorous brain tissues. Notably, paxillin overexpression was significantly associated with the grade of malignancy $(P<0.05)$. Moreover, the enforced expression of paxillin promoted the migration and invasion of glioma cells, while the loss of paxillin expression efficiently suppressed cell migration and invasion of glioma cell lines.

Conclusion: Our data suggest that paxillin may function as an oncogene and its overexpression may be closely correlated with tumor progression of human gliomas by modulating tumor cell motility, implying the potential of paxillin as a new therapeutic target for glioma intervention.

Keywords: paxillin, glioma, progression, migration, invasion

\section{Introduction}

Human gliomas, the most common type of primary intracranial tumor, arise from the glial cells of the brain and constitute $\sim 30 \%$ of all brain and central nervous system tumors. ${ }^{1}$ Based on the classification of the World Health Organization (WHO), gliomas range from grade I (low-proliferative noninvasive tumors) up to grade IV (cytologically malignant, highly infiltrative, mitotically active and necrosis-prone glioblastoma $[\mathrm{GBM}]) .^{2}$ Due to its poor prognosis and direct repercussions on patient cognitive function and quality of life, GBM has been indicated as the most malignant histological type of gliomas. ${ }^{3}$ Current treatment of gliomas include surgery, radiotherapy and chemotherapy. ${ }^{4}$ However, the prognosis in patients with gliomas remains poor, mainly because of tumor recurrence after surgical removal and therapeutic resistance. ${ }^{5}$ Therefore, it is of great significance to clarify the underlying mechanisms that regulate tumor growth and metastasis of gliomas to identify novel therapeutic targets and the development of efficient therapeutics for human gliomas.

Paxillin, together with Hic-5 and leupaxin, belongs to the paxillin family of intracellular scaffold proteins, which play important roles in coordinating these signaling 
events downstream of integrin-extracellular matrix (ECM) ligation. ${ }^{6,7}$ Functionally, paxillin acts as a molecular adaptor or a scaffold protein. It comprises various discrete protein binding modules, many of which have been found in tumor samples and/or cancer cell lines. ${ }^{8}$ Paxillin coordinates and integrates multiple signals from the growth factors and cell surface receptors. ${ }^{9}$ Through these molecular interactions, it can modulate various pathological processes, such as cell growth, cell invasion, cell migration and tissue remodeling. ${ }^{10,11}$ An increasing number of studies have provided insight into the functions of paxillin in tumor development and aggressive progression. However, its involvement in human gliomas remains unclear. Therefore, we examined the expression patterns of paxillin gene and protein using quantitative real-time reverse transcription polymerase chain reaction (RT-qPCR), Western blot and immunohistochemistry analyses, respectively, in 120 pairs of glioma and matched nontumorous brain tissues. The associations between paxillin expression and various histopathological features of glioma patients were also statistically evaluated. Then, the functions of paxillin in cellular motility of glioma cells were determined using transwell assays in vitro.

\section{Patients and methods}

\section{Patients and tissue samples}

The present study was authorized by the Research Ethics Committee of The First Hospital of Huai'an City Affiliated to Nanjing Medical University, China. All the patients provided written informed consent for use of tumor tissues for clinical research, and the Ethical Committee of The First Hospital of Huai'an City Affiliated to Nanjing Medical University approved the research protocol. All specimens were handled and made anonymous according to the ethical and legal standards.

A total of 120 pairs of glioma and matched nontumorous brain samples were obtained from the Department of Neurology, the First Hospital of Huai' an City Affiliated to Nanjing Medical University, from January 2008 to December 2012. The patient population consisted of 120 adults (80 males and 40 females; mean age at sampling of 56.2 years). The patients' clinical characteristics, such as age, gender, tumor size, Karnofsky performance score (KPS) and WHO grade, collected for statistical analyses are summarized in Table 1. For immunohistochemistry analysis, the resected glioma and nontumorous brain tissues were fixed in formalin, embedded in paraffin and cut into $4 \mu \mathrm{m}$ thick sections. For RT-qPCR and Western blot analyses, tissues were immediately frozen in liquid nitrogen and kept at $-80^{\circ} \mathrm{C}$ until analysis.
Table I Association of paxillin protein expression with different histopathological features of glioma patients

\begin{tabular}{|c|c|c|c|c|}
\hline $\begin{array}{l}\text { Histopathological } \\
\text { features }\end{array}$ & $\begin{array}{l}\text { No of } \\
\text { cases (\%) }\end{array}$ & $\begin{array}{l}\text { Paxillin- } \\
\text { high, n (\%) }\end{array}$ & $\begin{array}{l}\text { Paxillin- } \\
\text { low, } \mathbf{n}(\%)\end{array}$ & $P$-value \\
\hline Age, years & & & & NS \\
\hline$<50$ & $62(5 \mathrm{I} .67)$ & $32(5 I .6 I)$ & 30 (48.9) & \\
\hline$\geq 50$ & $58(48.33)$ & $30(5 \mathrm{I} .72)$ & $28(48.28)$ & \\
\hline Gender & & & & NS \\
\hline Male & $80(66.67)$ & $40(50.00)$ & $40(50.00)$ & \\
\hline Female & $40(33.33)$ & $22(55.00)$ & $18(45.00)$ & \\
\hline KPS & & & & NS \\
\hline$\geq 80$ & $68(56.67)$ & $37(54.4 I)$ & 31 (45.59) & \\
\hline$<80$ & $52(43.33)$ & $25(48.08)$ & $27(51.92)$ & \\
\hline WHO grade & & & & $<0.05$ \\
\hline I & $30(33.33)$ & $5(16.67)$ & $25(83.33)$ & \\
\hline II & $30(33.33)$ & $10(33.33)$ & $20(66.67)$ & \\
\hline III & $25(20.83)$ & $17(68.00)$ & $8(32.00)$ & \\
\hline IV & $35(29.17)$ & 30 (85.7I) & 5 (14.29) & \\
\hline Metastasis status & & & & $<0.05$ \\
\hline No & 91 (75.83) & $37(40.66)$ & $54(59.34)$ & \\
\hline Yes & $29(24.17)$ & $25(86.21)$ & 4 (I3.79) & \\
\hline
\end{tabular}

Note: "NS" refers to the difference without statistical significance. Abbreviations: KPS, Karnofsky performance score; WHO, World Health Organization.

\section{Cell culture and transfection}

Human glioma cell lines U251 and U87 were purchased from the cell bank of the Chinese Academy of Science. The cells were cultured in Dulbecco's Modified Eagle's Medium (DMEM) supplemented with 10\% fetal bovine serum (FBS, Life Technologies Corporation, Carlsbad, CA, USA), $100 \mathrm{U} / \mathrm{mL}$ of penicillin (Life Technologies Corporation) and streptomycin (Life Technologies Corporation) in a humidified atmosphere of $5 \% \mathrm{CO}_{2}$ at $37^{\circ} \mathrm{C}$.

To increase the expression of endogenous paxillin, its coding sequence was amplified and subcloned into the pcDNA3.1 (+) vector (Invitrogen, Carlsbad, CA, USA) based on the manufacturer' instructions. To knockdown the expression of endogenous paxillin, small interfering RNA (siRNA) duplex oligonucleotides targeting human paxillin mRNA (si-paxillin) was obtained from Ribobio (Guangzhou, China). The above-mentioned vectors were transiently transfected using Lipofectamine 2000 based on the instruction of the manufacturers (Invitrogen).

\section{RNA extraction and RT-qPCR}

Total RNA in tissues and cells was isolated using TRIzol ${ }^{\mathrm{TM}}$ Reagent (Invitrogen) according to manufacturers' instructions. Reverse transcription (RT) reactions were performed using the Prime-Script RT reagent kit (TaKaRa, Dalian, China). The qPCR was performed using a SYBR Premix Ex Taq TM II kit (TaKaRa). Glyceraldehyde 3-phosphate 
dehydrogenase (GAPDH) was used as an internal control. Real-time PCR was performed using an ABI PRISM 7500 Fast Real-time PCR System (Applied Biosystems, CA, USA). The following were the PCR primer sequences: paxillin forward: 5'-ACG TCT ACA GCT TCC CCA ACA A-3'; paxillin reverse: 5'-AGC AGG CGG TCG AGT TCA-3'; GAPDH forward: 5'-CAT GAC CAC AGT CCA TGC CAT CAC T- $3^{\prime}$ and GAPDH reverse: $5^{\prime}$-TGA GGT CCA CCA CCC TGT TGC TGT A-3'. Data were analyzed by the relative quantification method $\left(2^{-\Delta \Delta c t}\right)$. All experiments were performed on three separate occasions.

\section{Western blot analysis}

Total proteins were extracted from tissues and cells using a modified RIPA buffer (150 mM NaCl, 1\% NP240, 0.5\% sodium deoxycholate, $0.1 \%$ sodium dodecyl sulfate (SDS) and $50 \mathrm{mM}$ Tris $\mathrm{pH}$ 7.4) in the presence of Complete Mini protease inhibitor cocktail (Roche Diagnostics $\mathrm{GmbH}$, Mannheim, Germany). Western blot analysis was performed after separation of $50 \mu \mathrm{g}$ of proteins on a SDS/4\%-20\% polyacrylamide gel (Bio-Rad, Hercules, CA, USA) with conventional methods and an enhanced chemiluminescence detection kit (Amersham, Piscataway, NJ, USA). After that, filters were probed with rabbit antihuman Paxillin antibody (1:500, Cell Signaling Technologies, Danvers, MA, USA) and rabbit antihuman GAPDH (1:1,000; Santa Cruz Biotechnology, USA) plus horseradish peroxidase (HRP)conjugated corresponding secondary antibodies at 1:1,000 (Cell Signaling Technologies). The densities of bands were measured using a computer-assisted image analysis system (Adobe Systems, San Jose, CA, USA) and were normalized against the expression level of GAPDH protein. All experiments were performed on three separate times.

\section{Immunohistochemistry}

Surgically resected glioma tissues were fixed with $10 \%$ formalin and embedded in paraffin, and $4 \mu \mathrm{m}$-thick specimen sections were prepared on glass slides. The slides were deparaffinized using a graded ethanol series, and endogenous peroxidase activity was blocked by soaking in $0.3 \%$ hydrogen peroxide. After retrieving the antigen, hydrogen peroxide $(0.3 \%)$ was applied to block endogenous peroxide activity for 20 minutes after cooling. Then, the sections were incubated with rabbit antihuman Paxillin antibody (diluted 1:500, Cell Signaling Technologies) for 4 hours at $25^{\circ} \mathrm{C}$. All sections were processed based on the peroxidase-antiperoxidase method (Dako, Hamburg, Germany). Then, the peroxidase reaction was visualized by incubating the slides with DAB.
After that, the slides were counterstained with hematoxylin, examined by light microscopy and mounted.

To assess the immunostaining results, two independent pathologists, who were both blinded to the patients' background, were invited to score paxillin protein immunostaining based on staining intensity and positive cells' percentage as described in previous studies. ${ }^{12,13}$ In brief, staining intensity was scored as follows: "0" negative, "1" weakly positive, " 2 " moderately positive, and " 3 " strongly positive. The percentage of paxillin-positive cells was scored as $0(0 \%)$, $1(1 \%-25 \%), 2(26 \%-50 \%)$ and $3(>50 \%)$. The immunoreactive score (IRS) of paxillin protein for each tissue section was calculated by multiplying the intensity and the percentage scores. The median value of IRS was used as a cutoff point to divide 120 glioma patients into the low paxillin expression group and the high paxillin expression group.

\section{Cell migration and cell invasion assays}

The migration and invasion abilities of human glioma cells transfected with pcDNA3.1-paxillin/NC or si-paxillin/ $\mathrm{NC}$ were tested by cell migration and cell invasion assays. For the cell migration assay, $5 \times 10^{4}$ cells in serum-free medium were seeded in the upper chamber of a Transwell apparatus (Corning, NY, USA). Medium containing 10\% FBS was added into the lower chamber as a chemoattractant. After 24 hours of incubation, the cells on the top surface of the insert were removed with a cotton swab, whereas the cells that migrated through the membrane to the lower side were fixed with $4 \%$ paraformaldehyde and stained with $0.1 \%$ crystal violet. The number of migrated cells on the lower side of the membrane was counted at $200 \times$ magnification from ten different fields per section. The cell invasion assay was performed according to the similar procedure to the cell migration assay, except that the Transwell membranes were precoated with $24 \mu \mathrm{g} / \mu \mathrm{L}$ Matrigel (R\&D Systems, USA). The number of invasive cells on the lower side of the membrane was counted at $200 \times$ magnification from ten different fields per section. All assays were independently repeated at least three times, and the data were shown as the mean value of cells in ten fields based on three independent experiments.

\section{Statistical analysis}

Statistical analyses in this study were performed using SPSS Version 11.0 for Windows (SPSS Inc, IL, USA). Data are presented as mean $\pm \mathrm{SD}$. The differences in the expression of paxillin mRNA and protein between glioma and nontumorous brain tissues were analyzed using paired $t$-test. The $\chi^{2}$ test or Fisher's exact test was performed to assess the association 
between the IRS of paxillin protein and the histopathological parameters of glioma patients. For migration and invasion assays, Student's $t$-test was used for comparison between groups. Differences among groups were regarded to be statistically significant if the $P$-value was $<0.05$.

\section{Results}

\section{Upregulation of paxillin gene and protein in human glioma tissues}

Compared to nontumorous brain tissues, paxillin gene expression (glioma vs nontumorous: $3.32 \pm 1.30$ vs $1.88 \pm 0.76$,
$P<0.05$, Figure 1A) was markedly upregulated in glioma tissues. Similarly, the expression of paxillin protein in glioma tissues was significantly higher than that in nontumorous brain tissues (glioma vs nontumorous: $2.67 \pm 1.09$ vs $1.76 \pm 0.66, P<0.05$, Figure $1 \mathrm{~B}$ ).

In addition, the immunostaining of paxillin protein was positively localized in the cytoplasm and membrane of tumor cells in human glioma tissues, but was weak or negative in nontumorous brain tissues (Figure 1C). Statistically, the IRS values of paxillin protein in human glioma tissues were markedly higher than those in nontumorous brain

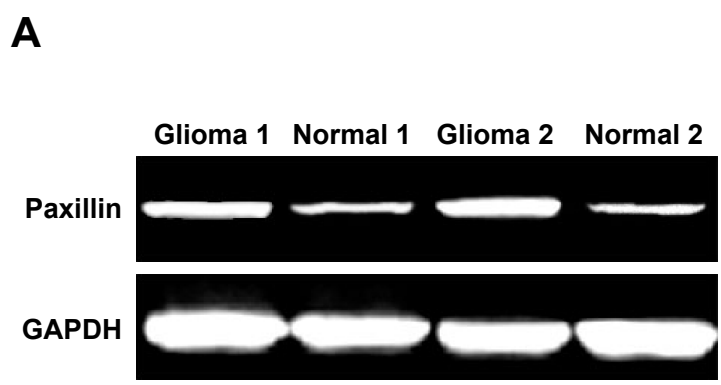

B

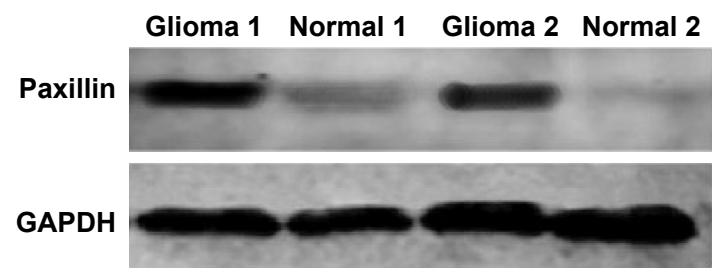

C

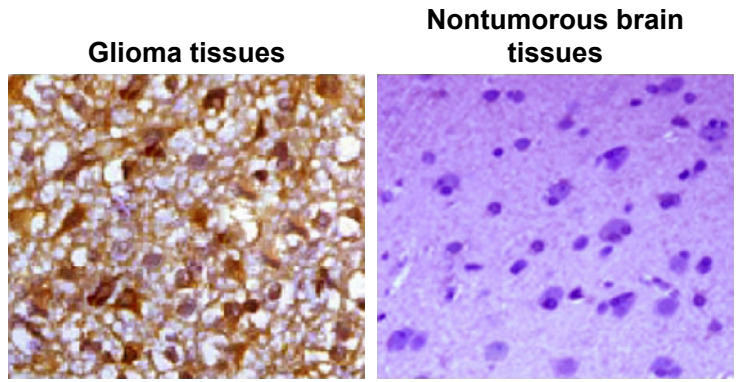

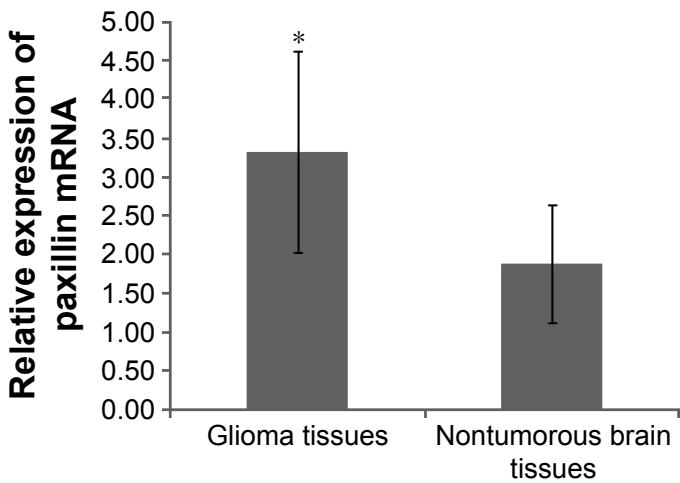
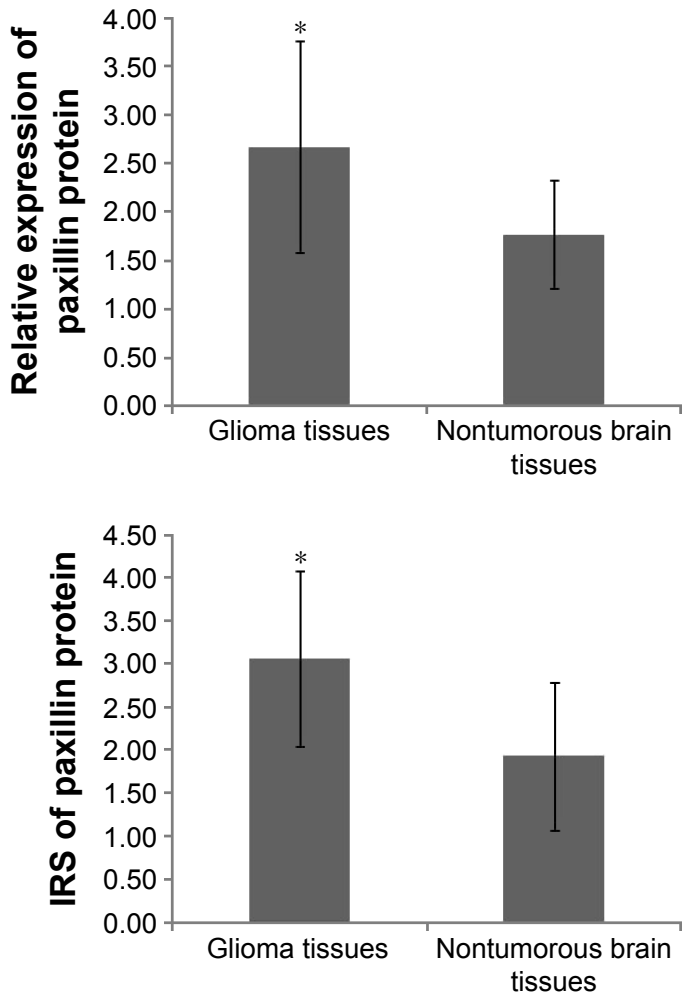

Figure I Upregulation of paxillin mRNA and protein in human glioma tissues.

Notes: (A) RT-qPCR analysis was performed to detect the expression levels of paxillin mRNA in glioma and nontumorous brain tissues. The paired $t$-test was used for this assay $(* P<0.05)$. (B) Western blot analysis was performed to detect the expression levels of paxillin protein in glioma and nontumorous brain tissues. The paired $t$-test was used for this assay $(* P<0.05)$. (C) Immunohistochemistry analysis showed that the immunostaining of paxillin protein was positively localized in the cytoplasm and membrane of tumor cells in human glioma tissues, but was weak or negative in nontumorous brain tissues (magnification $\times 200$ ). The paired $t$-test was used for this assay $(* P<0.05$ ). Abbreviations: RT-qPCR, real-time reverse transcription polymerase chain reaction; GAPDH, glyceraldehyde 3-phosphate dehydrogenase; IRS, immunoreactive score. 
A

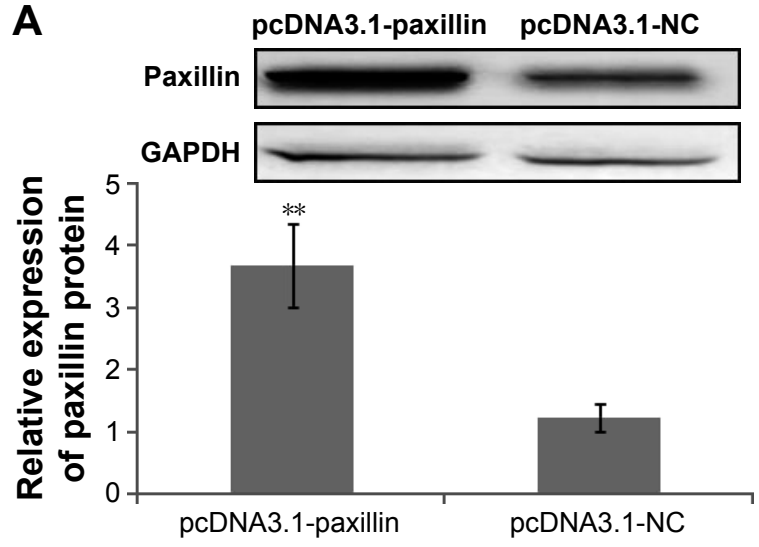

C

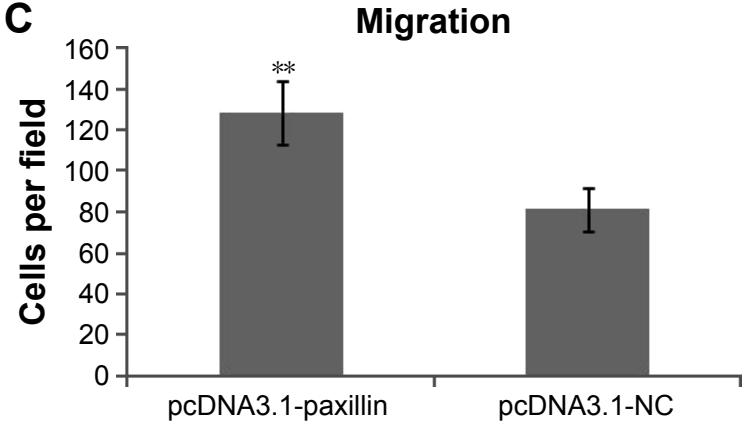

B

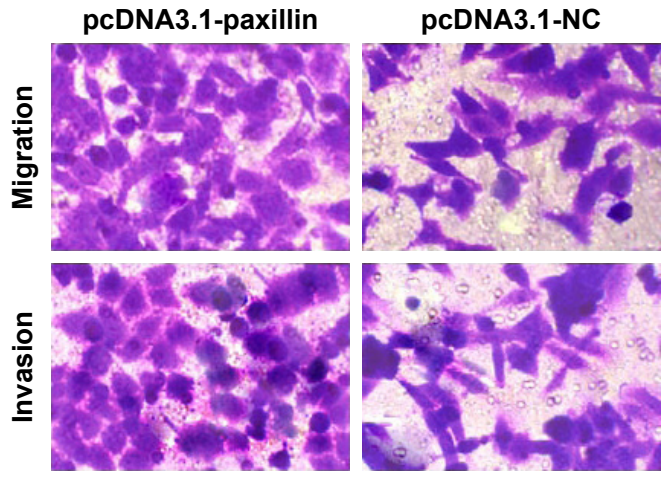

D

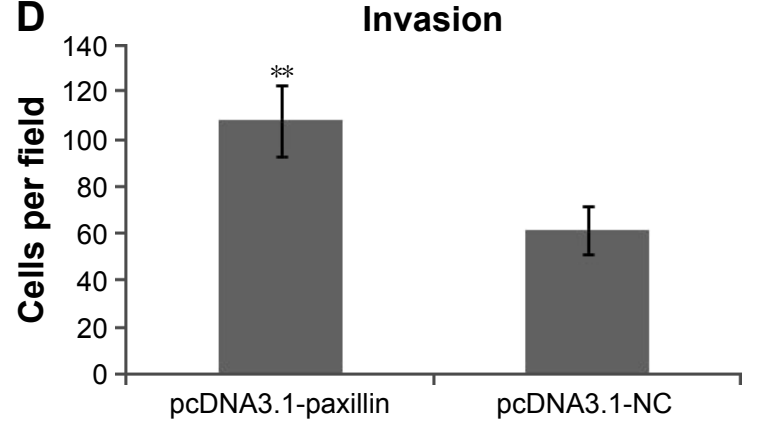

Figure 2 Enforced expression of paxillin promotes migration and invasion of glioma cells in vitro.

Notes: (A) Western blot analysis was performed to detect the expression levels of paxillin protein in U25I cells transfected with pcDNA3.I-paxillin and pcDNA3.I-NC. The independent $t$-test was used for this assay $(* * P<0.01)$. (B-D) The migration and invasion abilities of human glioma cells transfected with pcDNA3.I-paxillin/NC were tested by cell migration and cell invasion assays. The independent $t$-test was used for this assay $(* * P<0.0 \mathrm{I})$.

Abbreviation: GAPDH, glyceraldehyde 3-phosphate dehydrogenase.

tissues (glioma vs nontumorous: $3.06 \pm 1.01$ vs $1.93 \pm 0.86$, $P<0.05$, Figure 1C).

\section{Paxillin protein upregulation associates with WHO grade of human gliomas}

To determine the associations of paxillin protein expression with various histopathological factors, 120 glioma patients were divided into the high paxillin expression group and the low paxillin expression group using its median IRS values in glioma tissues as a cutoff point. Of 120 glioma patients, 62 (51.67\%) cases belonged to the high paxillin expression group and 58 (48.33\%) cases belonged to the low paxillin expression group. As shown in Table 1, glioma patients with advanced WHO grade more frequently had higher paxillin expression than those with early WHO grade $(P<0.05)$. Moreover, the expression of paxillin protein was significantly associated with tumor metastasis status of glioma tissues $(P<0.05$, Table 1$)$.

\section{Paxillin functions as an oncogene protein by promoting cell migration and cell invasion of glioma cells in vitro}

To further explore the functions of paxillin in the malignant phenotypes of glioma cells, both U251 and U87 cells were transfected with pcDNA3.1-paxillin/ $\mathrm{NC}$ or si-paxillin/NC. As a result, the expression levels of paxillin protein in U251 and U87 cells transfected with pcDNA3.1-paxillin were significantly higher than those transfected with pcDNA3.1-NC $(P<0.05$, Figure $2 \mathrm{~A}$ for $\mathrm{U} 251$ and Figure S1A for U87), while the transfection of si-paxillin efficiently reduced the expression levels of paxillin protein in U251 and U87 cells $(P<0.05$, Figure $3 \mathrm{~A}$ for $\mathrm{U} 251$ and Figure S2A for U87).

Then, we found that the enforced expression of paxillin dramatically promoted $(P<0.05$, Figure $2 \mathrm{~B}-\mathrm{D}$ for $\mathrm{U} 251$ and Figure S1B-D for U87), but the loss of paxillin dramatically retarded cell migration and invasion of $\mathrm{U} 251$ cells $(P<0.05$, Figure 3B-D for U251 and Figure S2B-D for U87).

\section{Discussion}

Human gliomas represent the most common malignancies characterized by rapid growth, frequent postoperative recurrence and poor prognosis. ${ }^{14,15}$ Despite the advancement of comprehensive treatments for these malignancies, including surgical resection, radiotherapy and chemotherapy, their therapeutic effects remain unsatisfactory. ${ }^{16,17}$ Therefore, a better clarification of the molecular mechanisms underlying tumor growth and invasion is required to improve the 
A

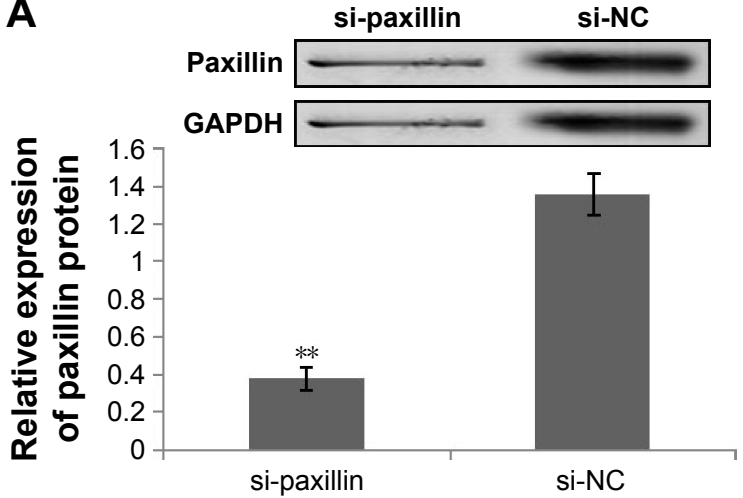

C

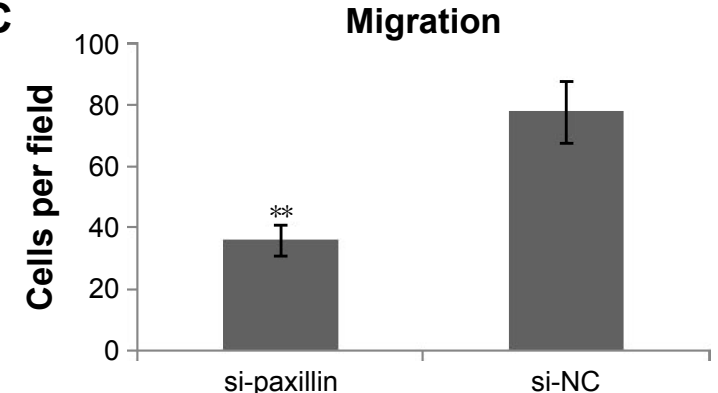

B

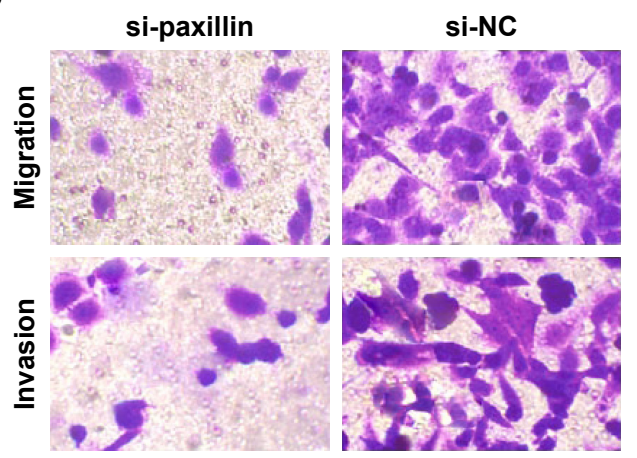

D Invasion

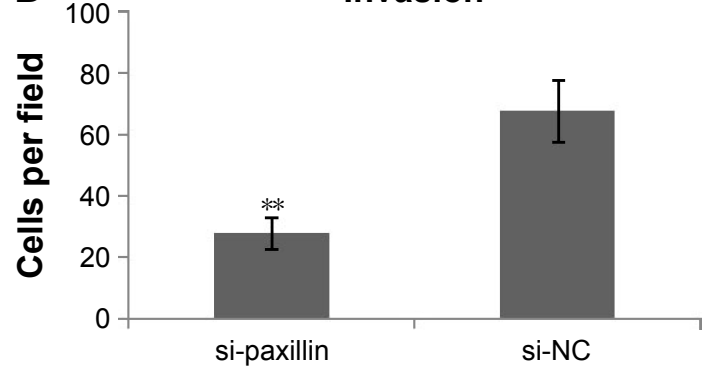

Figure 3 Loss of paxillin suppresses migration and invasion of glioma cells in vitro.

Notes: (A) Western blot analysis was performed to detect the expression levels of paxillin protein in U25I cells transfected with si-paxillin and si-NC. The independent $t$-test was used for this assay $(* * P<0.0 I)$. (B-D) The migration and invasion abilities of human glioma cells transfected with si-paxillin/NC were tested by cell migration and cell invasion assays. The independent $t$-test was used for this assay $(* * P<0.0 \mathrm{I})$.

Abbreviations: GAPDH, glyceraldehyde 3-phosphate dehydrogenase; si-NC, small-inference normal control.

therapeutic efficiency of glioma treatment. In the present study, RT-qPCR, Western blot and immunohistochemistry analyses showed the increased levels of paxillin mRNA and protein expression in glioma tissues compared to matched nontumorous brain tissues. Notably, high paxillin expression was significantly associated with the advanced tumor and the presence of metastasis. Moreover, the enforced expression of paxillin promoted motility of glioma cells, while the loss of paxillin expression efficiently inhibited motility of glioma cells. These findings not only confirm the oncogenic role of paxillin but also imply that paxillin might function as a potential therapeutic target for human gliomas.

Cell adhesion to neighboring cells, the surrounding ECM or stroma has been indicated to be crucial during the progression via regulating tumor cell proliferation and survival as well as enabling tumor cell dissemination and metastasis. ${ }^{18,19}$ Growing evidence show that the paxillin family of intracellular scaffold proteins may play roles in regulating the cellular migration machinery by coordinating multiple signals from growth factors, integrins, and cell surface receptors. ${ }^{20,21}$ As a member of the paxillin family, the paxillin gene, encoding for a focal adhesion molecule of $68 \mathrm{kD}$, was originally identified as a tyrosine-containing protein in cells transformed by the src oncogene. ${ }^{10,22}$ Functionally, paxillin regulates various biological processes, such as cell growth, cell motility and metastasis, matrix organization and tissue remolding. ${ }^{20} \mathrm{In}$ addition to the interactions with cytoskeleton proteins, paxillin can also bind to several oncogenic proteins, such as v-Src, E6 and BCR-ABL. ${ }^{23}$ Accumulating studies have reported that paxillin may be implicated into the pathogenesis of diverse human malignancies. For example, German et $\mathrm{al}^{24}$ showed that paxillin could promote tumor angiogenesis by regulating the expression of neuropilin 2; Mackinnon et $\mathrm{al}^{25}$ found the overexpression of paxillin in premalignant areas of hyperplasia, squamous metaplasia and goblet cell metaplasia, as well as dysplastic lesions and carcinoma in high-risk patients; $\mathrm{Wu}$ et $\mathrm{al}^{26,27}$ further reported that paxillin upregulation induced by miR-218 suppression might be an independent predictor of survival and relapse in nonsmall cell lung cancer and oral cavity squamous cell carcinoma; Xiao et a ${ }^{28}$ reported that paxillin was strongly expressed in gastric adenoma compared with that in nonneoplastic mucosa and carcinoma, and also found that the aberrant expression of paxillin might be implicated into the differentiation, growth and metastasis of gastric carcinoma and Sen et $\mathrm{al}^{29}$ indicated that paxillin regulated prostate cancer proliferation by serving as a mediator between extranuclear kinase signaling and intranuclear transcriptional signals. Consistent with these previous findings, our data 
revealed the overexpression of paxillin mRNA and protein in human glioma tissues compared to that in normal brain tissues and found an increasing tendency of paxillin expression from grade I to grade IV. Statistically, the expression level of paxillin protein was positively correlated with the advanced WHO grades and the presence of metastasis. These findings suggest the crucial roles of paxillin in tumorigenesis and aggressive progression of human gliomas.

Since the association between paxillin protein overexpression and positive metastasis of glioma patients was significant, we hypothesized that paxillin might promote cell motility of glioma cells. To address this hypothesis, we enhanced and reduced the expression of paxillin protein in both U251 and U87 cells, respectively, by transfecting pcDNA3.1 vector and RNA interference specific to paxillin. Functionally, we observed that the enforced expression of paxillin promoted the motility of glioma cells, implying its oncogenic roles, which might be suppressed by decreasing the expression of paxillin.

Although we first revealed the clinical relevance and biological functions of paxillin deregulation in human gliomas, there is a limitation in the present study. Accumulating studies have investigated the underlying mechanisms of paxillin in carcinogenesis and cancer progression of various malignancies. ${ }^{24,26,27,29}$ However, the mechanism of action on how paxillin expression impacts human gliomas remains unclear. More studies on this point are required in the future.

\section{Conclusion}

Our data suggest that paxillin may function as an oncogene and its overexpression may be closely correlated with tumor progression of human gliomas by modulating tumor cell motility, implying the potential of paxillin as a novel therapeutic target for glioma intervention.

\section{Disclosure}

The authors report no conflicts of interest in this work.

\section{References}

1. Cohen AL, Colman H. Glioma biology and molecular markers. Cancer Treat Res. 2015;163:15-30.

2. Morokoff A, Ng W, Gogos A, Kaye AH. Molecular subtypes, stem cells and heterogeneity: implications for personalised therapy in glioma. J Clin Neurosci. 2015;22(8):1219-1226.

3. Rizzo D, Ruggiero A, Martini M, Rizzo V, Maurizi P, Riccardi R. Molecular biology in pediatric high-grade glioma: impact on prognosis and treatment. Biomed Res Int. 2015;2015:215135.

4. Ma C, Zhao G, Cruz MH, Siden A, Yakisich JS. Translational gap in glioma research. Anticancer Agents Med Chem. 2014;14(8):1110-1120.

5. Diamond EL, Corner GW, De Rosa A, Breitbart W, Applebaum AJ. Prognostic awareness and communication of prognostic information in malignant glioma: a systematic review. J Neurooncol. 2014;119(2): $227-234$.
6. Mazaki Y, Hashimoto S, Sabe H. Monocyte cells and cancer cells express novel paxillin isoforms with different binding properties to focal adhesion proteins. J Biol Chem. 1997;272(11):7437-7444.

7. Salgia R, Li JL, Lo SH, et al. Molecular cloning of human paxillin, a focal adhesion protein phosphorylated by $\mathrm{P} 210 \mathrm{BCR} / \mathrm{ABL}$. J Biol Chem. 1995;270(10):5039-5047.

8. Hagel M, George EL, Kim A, et al. The adaptor protein paxillin is essential for normal development in the mouse and is a critical transducer of fibronectin signaling. Mol Cell Biol. 2002;22(3):901-915.

9. Turner CE, Glenney JR Jr, Burridge K. Paxillin: a new vinculinbinding protein present in focal adhesions. J Cell Biol. 1990;111(3): 1059-1068.

10. Brown MC, Turner CE. Paxillin: adapting to change. Physiol Rev. 2004;84(4):1315-1339.

11. Turner CE. Paxillin interactions. J Cell Sci. 2000;113(pt 23):4139-4140.

12. Li M, Li X, Xu S, et al. Protein phosphatase 4 catalytic subunit is overexpressed in glioma and promotes glioma cell proliferation and invasion. Tumour Biol. Epub 2016 Apr 9.

13. Sun J, Zhang L, Zhao H, et al. CHD1L regulates cell cycle, apoptosis, and migration in glioma. Cell Mol Neurobiol. 2016;36(4):565-576.

14. Ohgaki H, Kleihues P. Epidemiology and etiology of gliomas. Acta Neuropathol. 2005;109(1):93-108.

15. Liu N, Tu Y. Systematic review of microRNAs and its therapeutic potential in glioma. Cancer Transl Med. 2015;1:50-66.

16. Cheng Y, Tu Y, Liang P. Promoter methylated tumor suppressor genes in glioma. Cancer Transl Med. 2015;1:123-130.

17. Barcellos-Hoff MH, Newcomb EW, Zagzag D, Narayana A. Therapeutic targets in malignant glioblastoma microenvironment. Semin Radiat Oncol. 2009;19(3):163-170.

18. Friedl P, Wolf K. Tumour-cell invasion and migration: diversity and escape mechanisms. Nat Rev Cancer. 2003;3(5):362-374.

19. Yu JA, Deakin NO, Turner CE. Paxillin kinase-linker tyrosine phosphorylation regulates directional cell migration. Mol Biol Cell. 2009; 20(22):4706-4719.

20. Schaller MD. Paxillin: a focal adhesion-associated adaptor protein. Oncogene. 2001;20(44):6459-6472.

21. Turner CE. Paxillin and focal adhesion signalling. Nat Cell Biol. 2000; 2(12):E231-E236.

22. Deakin NO, Turner CE. Paxillin comes of age. J Cell Sci. 2008; 121(pt 15):2435-2444.

23. Sattler M, Pisick E, Morrison PT, Salgia R. Role of the cytoskeletal protein paxillin in oncogenesis. Crit Rev Oncog. 2000;11(1):63-76.

24. German AE, Mammoto T, Jiang E, Ingber DE, Mammoto A. Paxillin controls endothelial cell migration and tumor angiogenesis by altering neuropilin 2 expression. J Cell Sci. 2014;127(pt 8):1672-1683.

25. Mackinnon AC, Tretiakova M, Henderson L, et al. Paxillin expression and amplification in early lung lesions of high-risk patients, lung adenocarcinoma and metastatic disease. J Clin Pathol. 2011;64(1):16-24.

26. Wu DW, Cheng YW, Wang J, Chen CY, Lee H. Paxillin predicts survival and relapse in non-small cell lung cancer by microRNA-218 targeting. Cancer Res. 2010;70(24):10392-10401.

27. Wu DW, Chuang CY, Lin WL, Sung WW, Cheng YW, Lee H. Paxillin promotes tumor progression and predicts survival and relapse in oral cavity squamous cell carcinoma by microRNA-218 targeting. Carcinogenesis. 2014;35(8):1823-1829.

28. Xiao LJ, Zhao EH, Zhao S, et al. Paxillin expression is closely linked to the pathogenesis, progression and prognosis of gastric carcinomas. Oncol Lett. 2014;7(1):189-194.

29. Sen A, De Castro I, Defranco DB, et al. Paxillin mediates extranuclear and intranuclear signaling in prostate cancer proliferation. J Clin Invest. 2012;122(7):2469-2481. 


\section{Supplementary materials}

A
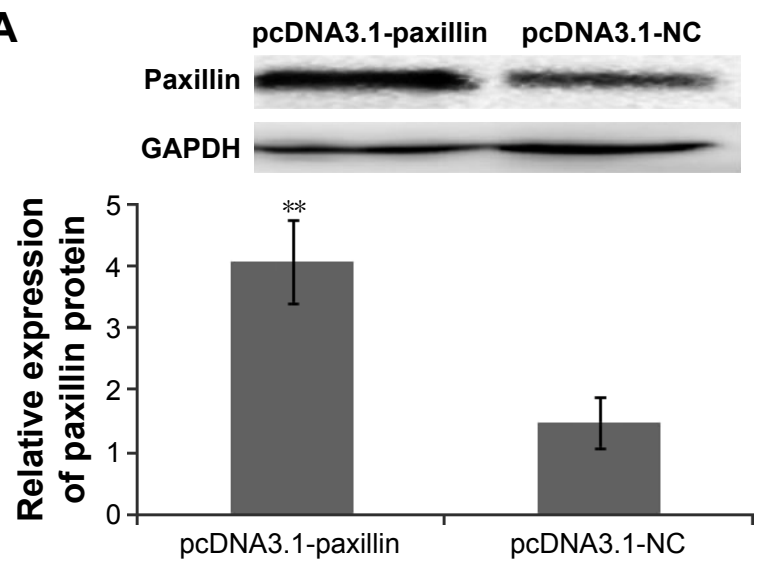

C

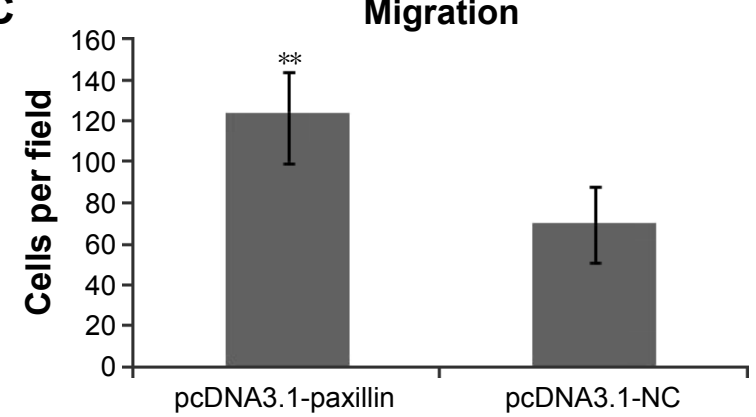

B
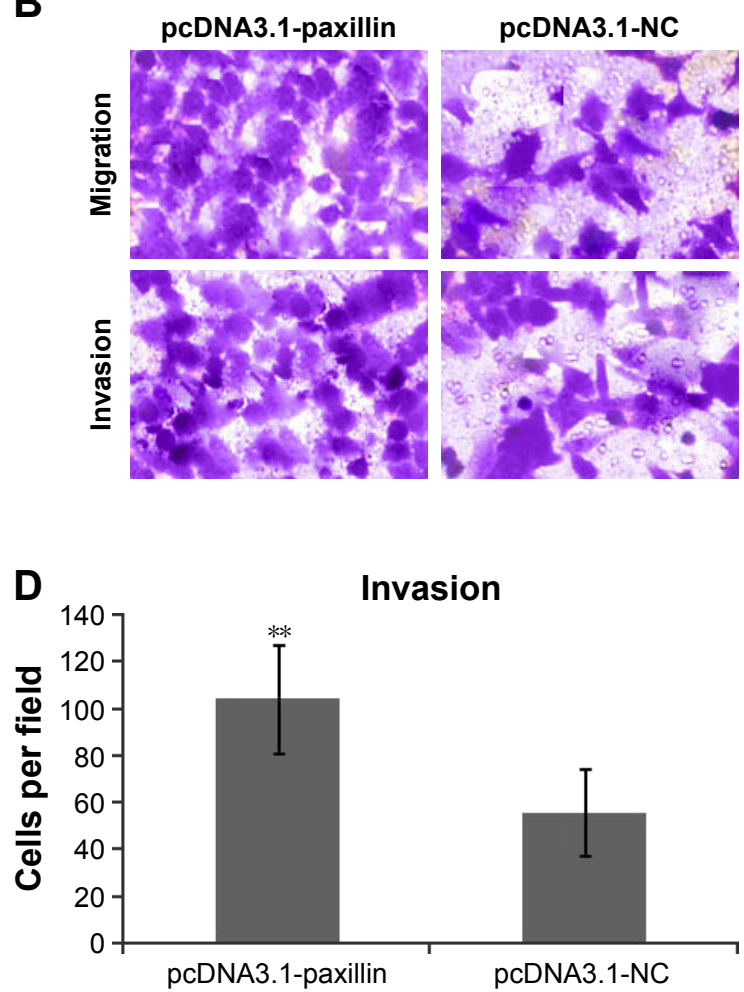

Figure SI Enforced expression of paxillin promotes migration and invasion of glioma cells in vitro.

Notes: (A) Western blot analysis was performed to detect the expression levels of paxillin protein in U87 cells transfected with pcDNA3. I-paxillin and pcDNA3. I-NC. The independent $t$-test was used for this assay $(* * P<0.01)$. (B-D) The migration and invasion abilities of human glioma cells transfected with pcDNA3.I-paxillin/NC were tested by cell migration and cell invasion assays. The independent $t$-test was used for this assay $(* * P<0.0 \mathrm{I})$.

Abbreviation: GAPDH, glyceraldehyde 3-phosphate dehydrogenase. 
A
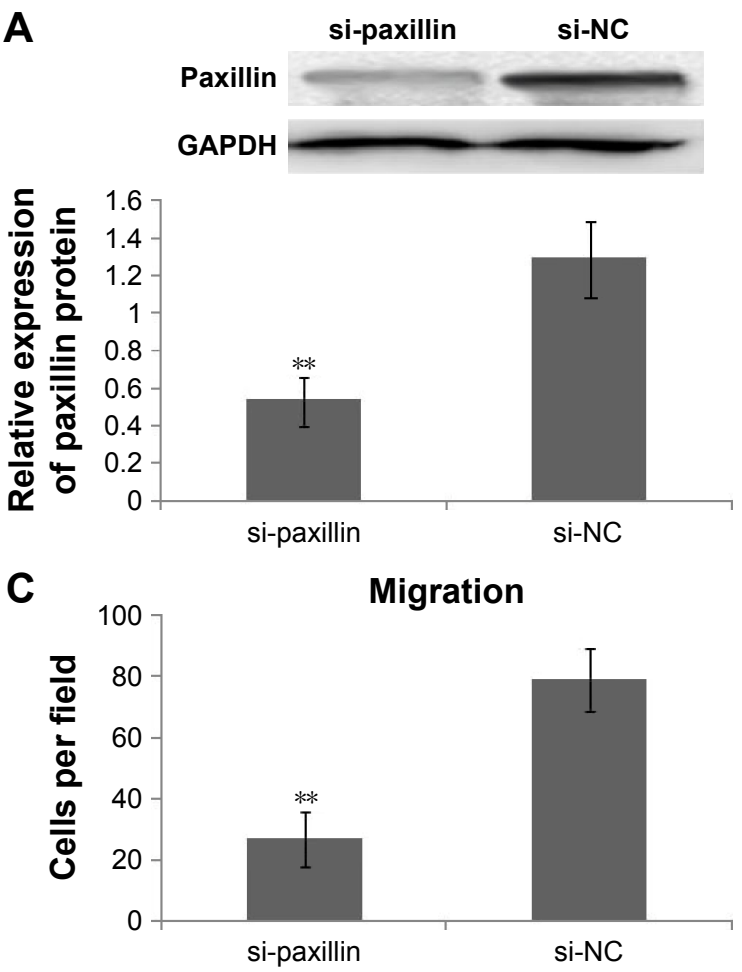

B

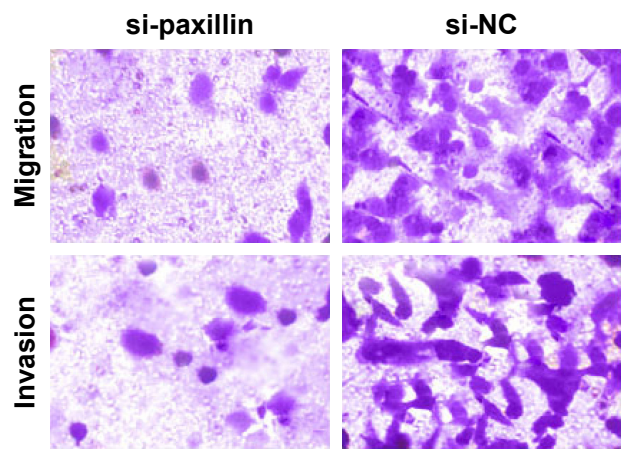

D

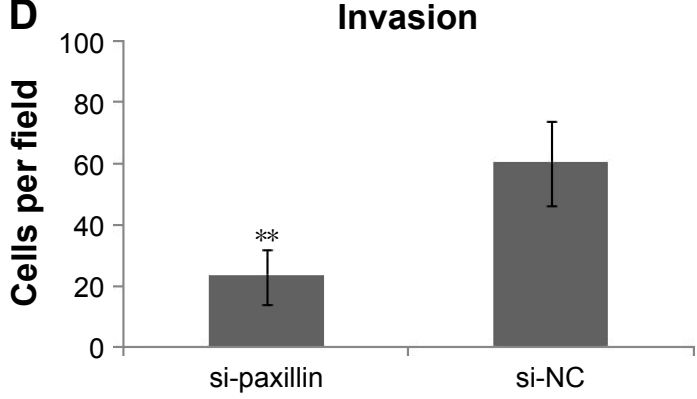

Figure S2 Loss of paxillin suppresses migration and invasion of glioma cells in vitro.

Notes: (A) Western blot analysis was performed to detect the expression levels of paxillin protein in U87 cells transfected with si-paxillin and si-NC. The independent $t$-test was used for this assay $(* * P<0.01)$. (B-D) The migration and invasion abilities of human glioma cells transfected with si-paxillin/NC were tested by cell migration and cell invasion assays. The independent $t$-test was used for this assay $(* * P<0.01)$.

Abbreviation: GAPDH, glyceraldehyde 3-phosphate dehydrogenase.

\section{Publish your work in this journal}

OncoTargets and Therapy is an international, peer-reviewed, open access journal focusing on the pathological basis of all cancers, potential targets for therapy and treatment protocols employed to improve the management of cancer patients. The journal also focuses on the impact of management programs and new therapeutic agents and protocols on

\section{Dovepress}

patient perspectives such as quality of life, adherence and satisfaction. The manuscript management system is completely online and includes a very quick and fair peer-review system, which is all easy to use. Visit http://www.dovepress.com/testimonials.php to read real quotes from published authors. 